\title{
DISEÑO DE UN BANCO DE PRUEBAS PARA EL ANÁLISIS DE VIBRACIONES MECÁNICAS
}

\section{DESIGN OF A TEST BANK FOR MECHANICAL VIBRATION ANALYSIS}

\author{
MSc. Ricardo Andrés García-León*, MSc. Eder Flórez Solano* \\ MSc. Jesús Pedroza *** \\ *Universidad Francisco de Paula Santander Ocaña, \\ Facultad de Ingenierías, Grupo de Investigación INGAP. \\ Vía Acolsure, Sede el Algodonal - Ocaña, Norte de Santander, Colombia. \\ Tel.: +57 5690088 Ext. 212 - 191. \\ E-mail: (ragarcial, enflorez) @ufpso.edu.co. \\ ** Universidad Francisco de Paula Santander, \\ Facultad de Ingenierías, programa de Ingeniería Mecánica. \\ Cúcuta, Norte de Santander. \\ E-mail: jpedroza@ufps.edu.co.
}

\begin{abstract}
Resumen: El análisis de las vibraciones mecánicas es considerado en el ámbito industrial como una técnica de mantenimiento, que contribuye en principio a detectar el comienzo de una futura avería o fallas ya desarrolladas y a la vez permite disponer de las herramientas necesarias para analizar la causa del problema que se está generando. Las vibraciones que ocurren en cualquier máquina rotativa y en sus estructuras circundantes, es el resultado de defectos mecánicos o de causas inherentes a la forma en que dicho equipo trabaja, es decir a su diseño y construcción. Analizar, comprender y conocer cuáles son las vibraciones que determinan el grado de defectuosidad mecánica y el origen de este efecto, representa y constituye el motivo por el cual se realizó un diseño para probar y conocer las vibraciones en los diferentes elementos mecánicos originadas por, desbalance, desalineamiento y daños en rodamientos; siendo estas tres, por lo general, la causa más frecuente de fallos de toda clase de maquinaria industrial y para la parte educativa con este proyecto lo que permite es estudiar la respuesta dinámica que cada componente ante estas manifestaciones y mejorar los elementos o sus posiciones.
\end{abstract}

Palabras clave: Vibraciones, Fallas, Maquina, Grietas, Norma.

\begin{abstract}
The analysis of mechanical vibrations is considered in the industrial sector as a technical maintenance, which contributes in principle to detect the start of a future breakdown or failure already developed and also makes available the tools needed to analyze the cause of the problem It is being generated. The vibrations that occur in any rotating machine and its surrounding structures, is the result of mechanical defects or causes inherent in the way the equipment works, ie its design and construction. Analyze, understand and know what the vibrations that determine the degree of mechanical malfunction and the origin of this effect are represented and is the why a design was carried out to try and meet the different vibrations caused by mechanical elements, imbalance , misalignment and bearing damage; being three, usually the most common cause of failure of all kinds of industrial machinery and for the educational part of this project which allows to study the dynamic response is that each component before these events and improve the elements or their positions.
\end{abstract}

Keywords: Vibrations, Faults, Machine, Cracks, Standard. 


\section{INTRODUCCIÓN}

El análisis de las vibraciones mecánicas es considerado en el ámbito industrial como una técnica de mantenimiento, que contribuye en principio a detectar el comienzo de una futura avería o fallas ya desarrolladas y a la vez permite disponer de las herramientas necesarias para analizar la causa del problema que se está generando. La mayoría de empresas utilizan este estudio para establecer el momento más oportuno para corregir eficaz y eficientemente cualquier problema detectado, ya que actualmente se considera esta tecnología predictiva como la mejor alternativa para evaluar el estado mecánico de toda máquina (García-León, Flórez-Solano, \& Sanchez-Ortiz, 2017) (FEM García, JJ Ramirez-Mateus, 2017).

Las vibraciones que ocurren en cualquier máquina rotativa y en sus estructuras circundantes, es el resultado de defectos mecánicos o de causas inherentes a la forma en que dicho equipo trabaja, es decir a su diseño y construcción, además estas vibraciones pueden proceder de una fuente externa, la cual se evita con la implementación de algunos materiales aislantes. Por esta razón y dado la naturaleza de las vibraciones este proyecto se encargará de analizar aquellas vibraciones que son el resultado de defectos mecánicos, debido a que estas pueden corregirse fácilmente con la implementación de técnicas adecuadas de mantenimiento y monitoreo de señales (E Florez, CAP Cortes 2017).

Analizar, comprender y conocer cuáles son las vibraciones que determinan el grado de defectuosidad mecánica y el origen de este efecto, representa y constituye el motivo por el cual se realizó este trabajo de grado, el cual está encaminado a diseñar un banco de pruebas que permita identificar las vibraciones originadas por, desbalance, desalineamiento y daños en rodamientos; siendo estas tres, por lo general, la causa más frecuente de fallos de toda clase de maquinaria industrial (Carrero-Blanco, Estrems, \& Sánchez, 2016) (JES Jaimes, JAV Ochoa, 2017).

Muchos ingenieros concentran sus esfuerzos en investigar la mejor forma de evitar la presencia incontrolable de vibraciones, que hace inminente un fallo catastrófico, debido al efecto en cadena que produce la proliferación de estas fuerzas dinámicas; pero este banco tratará de reproducir estas cargas indeseables para luego estudiarlas y de alguna manera llegar a comprenderlas (García-León, Flórez-Solano, \& Escobar-Macea, 2016).

La medición de estas señales vibratorias permite diagnosticar muchos problemas que se presentan durante el funcionamiento de cualquier pieza mecánica, hasta el punto de llegar predecir su posible falla, decir que está mal o que corregir. Sin embargo, analizar e interpretar estos resultados, es solo una iniciativa que promueve el diseño de este banco de pruebas, debido a que determinar o identificar la causa de un posible fallo requiere de experiencia y un buen entrenamiento en esta área.

Teniendo en cuenta lo anterior, la presente investigación busca el diseño óptimo de una máquina para la medición de vibraciones mecánicas que permita el desarrollo de experimentos basados en el fenómeno de agrietamiento, mediante teorías y cálculos de diseño mecánico.

\section{MATERIALES Y MÉTODOS}

En la figura 1, se observa el diseño inicial del banco de pruebas que permitirá el análisis de vibraciones mecánica y el cual estará constituido por un motor que se encargará de transmitir la potencia y velocidad al sistema, este se encontrará acoplado a un eje que llevará consigo un volante con agujeros roscados, la ubicación adecuada de estos orificios hace posible obtener vibraciones de desbalance mediante la instalación de tornillos prisioneros. Los soportes estarán dotados de tornillos para desalinear los ejes y así conseguir las vibraciones para cada tipo de desalineamiento; se debe aclarar que la desalineación provocada por estos tornillos tendrán que limitarse al permitido en los rodamientos y el acople. Las vibraciones originadas por defectos en rodamiento se obtienen con mucha facilidad, ya que simplemente se desmonta el rodamiento no defectuoso y luego se instala aquel que tenga algún tipo de falla plenamente identificada. Una vez, que el sistema esté funcionando con las características deseadas los sensores ubicados en cada soporte recibirán la señal, las cuales serán llevas a un registrador para luego ser analizadas (García-León, Flórez-Solano, \& Acosta, 2015) (Advanced Antivibration Components, 2004) (Dytran Instruments INC, 2007). 


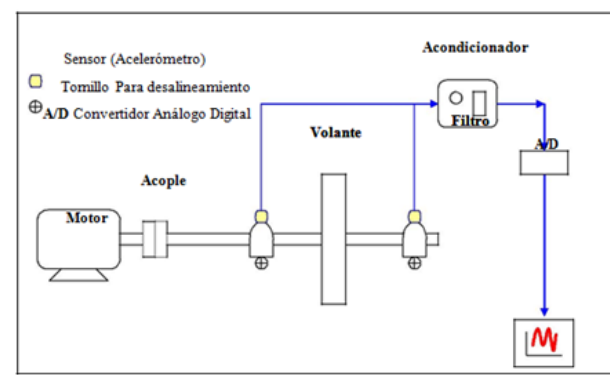

Fig. 1. Diseño inicial del banco de pruebas.

\section{RESULTADOS Y DISCUSIÓN}

Con la finalidad de obtener un correcto diseño de la maquina se llevaron a cabo cada uno de los siguientes cálculos:

\subsection{Diseño y cálculo de los elementos mecánicos}

Primero se selecciona el motor que no tiene mucha carga con las siguientes característica. Tensión conmutable 220/440V. Arranque directo 220V o $440 \mathrm{~V}$. Arranque estrella triángulo a $220 \mathrm{~V}$ o $440 \mathrm{~V}$.

- Aislamiento clase F tropicalizado.

- Totalmente cerrados enfriado con ventilación.

- Accionados con variador de velocidad sin pérdida de potencia.

\subsection{Longitud del eje y ubicación de rodamientos}

Antes de calcular el tamaño (diámetro) adecuado del eje el cual soportará los volantes, hay que determinar su longitud, ya que son muchas las variables que deben intervenir para obtener una dimensión apropiada; la ubicación relativa de los rodamientos y el acople es muy importante para conseguir el desalineamiento permisible de estos elementos. Además, se obtendrá el avance del tornillo que moverá los soportes del eje. El banco de pruebas debe permitir los tres tipos de desalineamiento angular, paralelo y combinación.

Desalineamiento angular. El esquema de la figura 28 muestra el desalineamiento angular esperado al trasladar solamente el cojinete rodante (A) a una nueva posición; la trayectoria seguida por este elemento es una curva con centro en el cojinete fijo (B), la cual está representada por la línea punteada (FA).

Debido a que el desplazamiento angular es muy pequeño se considera (FA) como una línea recta para facilitar el análisis, lo mismo sucede con el desplazamiento del acople (C). El objetivo de este razonamiento es poder determinar un desplazamiento aproximado para cualquier ángulo del cojinete rodante, que permita obtener el avance del tornillo de desalineamiento.

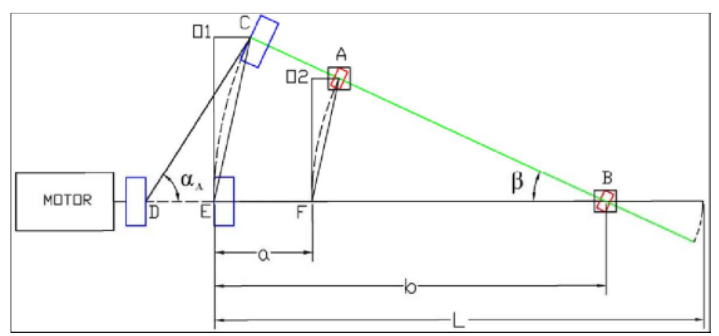

Fig. 2. Desalineamiento angular entre ejes.

Además, en la figura 2 se presenta el esquema que permite relacionar las características de acoplamiento, como por ejemplo la desalineación angular $(\alpha \mathrm{A})$ y paralelo $(\mathrm{O} 1 \mathrm{E})$, las cuales depende de la separación entre el acople conductor y conducido (DE). Aunque se desconoce el tamaño del eje, el análisis de esta figura contribuirá a determinar el tipo de cojinete rodante y acople que serán usados para el correcto funcionamiento del banco de pruebas.

Las dimensiones (O2F) y (O1E) son los desplazamientos verticales del movimiento en el rodamiento (A) y el Acople (C) respectivamente. $(\mathrm{O} 2 \mathrm{~F})$ se considera como el desplazamiento del soporte del rodamiento (A), esta aproximación se hace debido a que en el diseño del banco la trayectoria que seguirá su soporte será una línea recta con movimiento angular relativo del rodamiento y eje.

\subsection{Diseño y cálculo del volante}

Para conseguir el espectro de vibraciones debido a desbalance, el banco de pruebas necesita dos discos o volantes para producir la fuerza inercial de desequilibrio. Estos elementos deben ser capaces de introducir los cuatros tipos de desbalances que son: estático, par, casi estático y dinámico; además deben desbalancearse y balancearse fácilmente, es decir añadir o retirar peso, esto se consigue con tornillos prisioneros debidamente calibrados y ubicados en la superficie del volante (González, Gonzalo. 2003).

Selección material del volante. Se empleará aluminio por sus propiedades de, bajo peso, buena resistencia a la corrosión, facilidad relativa de formado y maquinado y apariencia agradable. Según las designaciones estandarizadas por la Asociación del Aluminio (AA, Aluminum Association) se escoge un aleación de aluminio tipo 
6061 T6, especificando sus propiedades en el siguiente cuadro.

Tabla 1. Aluminio tipo 6061 T6

\begin{tabular}{|c|c|c|c|c|}
\hline ALEACIÓN & TRATAMIENTO & $\begin{array}{l}\text { PROPIEDADES } \\
\text { MECÁNICAS }\end{array}$ & $\begin{array}{l}\text { PROPIEDADES } \\
\text { FÍSIIAS } \\
\text { QÚMICAS }\end{array}$ & $\begin{array}{l}\text { APLICACIONES } \\
\text { Y FORMAS }\end{array}$ \\
\hline $\begin{array}{l}6061 \\
\text { Magnesi } \\
\text { o } \\
\text { Silicio }\end{array}$ & $\begin{array}{l}\text { T6 } \\
\text { Tratamiento } \\
\text { Térmico por } \\
\text { Solución y } \\
\text { luego con } \\
\text { envejecimie } \\
\text { nto } \\
\text { artificial }\end{array}$ & 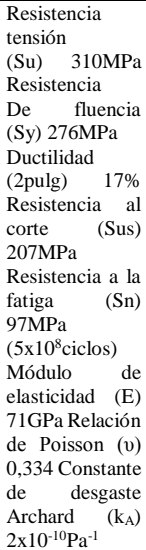 & $\begin{array}{l}\text { Densidad } 2732 \\
\mathrm{Kg} / \mathrm{m}^{3} \text { Buena } \\
\text { resistencia a la } \\
\text { corrosión, pero } \\
\text { las sustancias } \\
\text { alcalinas son } \\
\text { corrosivas para } \\
\text { el aluminio No } \\
\text { magnético } \\
\text { Buena } \\
\text { Conductividad }\end{array}$ & $\begin{array}{l}\text { Se emplea en } \\
\text { estructuras, } \\
\text { armazones, y } \\
\text { piezas de } \\
\text { vehículos, } \\
\text { usos marinos } \\
\text { Se consigue } \\
\text { en todas las } \\
\text { formas } \\
\text { (Barras, } \\
\text { tubo, etc.) }\end{array}$ \\
\hline
\end{tabular}

Fuente: MOTT, Robert. Diseño de elementos de máquinas. México: Prentice Hall, 2006. p.15

Geometría del volante. Analizando los cuatro tipos de desequilibrio como también los métodos generalmente utilizados para el balanceo, los discos deberán tener dos tipos de agujeros. El primer tipo corresponde a 8 agujeros de $14 \mathrm{~mm}$ ubicados a un radio de $75 \mathrm{~mm}$ desde el centro del disco y separados cada uno respectivamente por un ángulo de $45^{\circ}$.

La ISO 2953 (Balancing Machines-Description and Evaluation) recomienda perforar 12 agujeros roscados igualmente espaciados para su método de balanceo, lo que equivale a $30^{\circ}$ por cada agujero. Se decide ubicar cada agujero del volante a $45^{\circ}$ ya que el objetivo del banco es originar cargas de desbalance, además este ángulo facilita el cálculo de la fuerza centrífuga y ayuda a identificar adecuadamente el desequilibrio.

El segundo tipo son 8 agujeros de $10 \mathrm{~mm}$ a un radio de $55 \mathrm{~mm}$ y separados cada uno respectivamente por un ángulo de $45^{\circ}$. Además el ancho y el diámetro serán $30 \mathrm{~mm}$ y $180 \mathrm{~mm}$ respectivamente (escogido de tamaños métricos preferentes norma ANSI B.21978(r1994)) Esto se representa esquemáticamente en la figura 3.

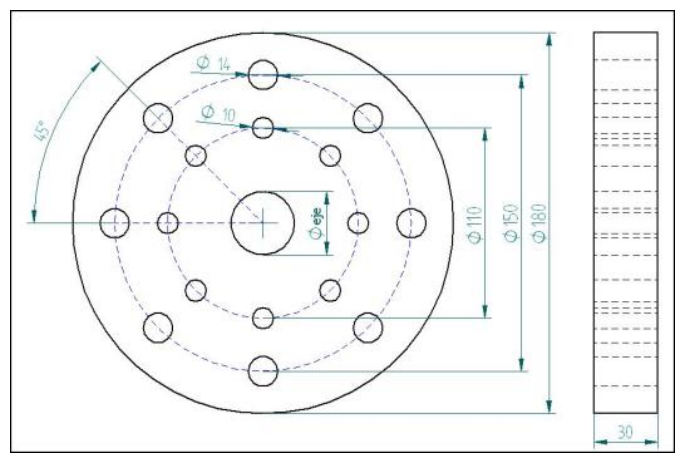

Fig. 3. Volante para el desbalance (medidas en $\mathrm{mm}$ ).

Con este volante se puede conseguir los cuatros tipo de desbalance anteriormente mencionados, ya que el ángulo de separación permite un desequilibrio dinámico al colocar o retirar un tornillo opresor en cualquier orientación, además con los tornillos de $10 \mathrm{~mm}$ se obtiene el desbalance casi estático.

Las figuras $4, \quad 5,6 \quad$ y 7 se muestran esquemáticamente cada tipo de desequilibrio, considerando que el disco se encuentra balanceado cuando no tienen ningún tornillo opresor, es decir que al colocar un tornillo se generarán cargas inerciales de desequilibrio.

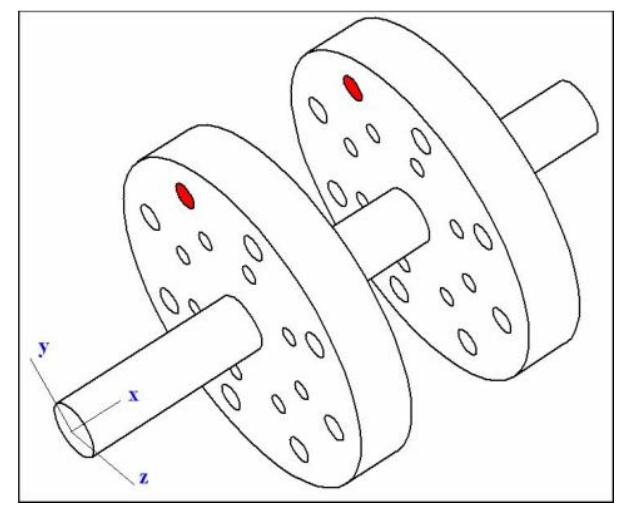

Fig. 4. Desbalance estático.

La figura 5 muestra el desbalance casi estático, donde el tornillo opresor (rojo) se instala en cada volante en sus agujeros de 14 y $10 \mathrm{~mm}$, pero de igual forma como aparece en la figura 32 estos tornillos se ubican en la misma dirección $\mathbf{y}$. 


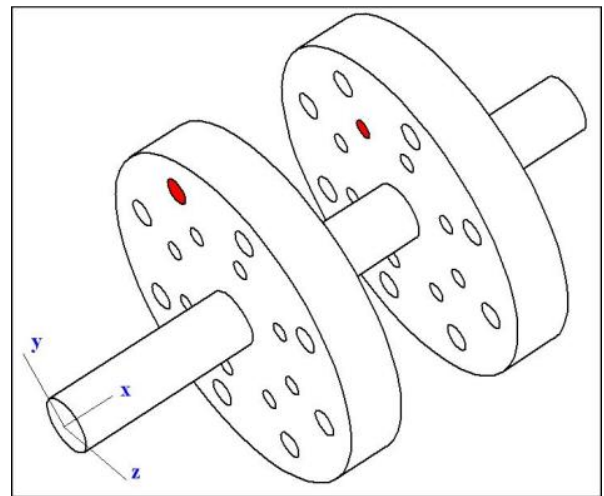

Fig. 5. Desbalance casi estático.

En la figura 6 se observa el desbalance par, donde en ambos volantes se instala el tornillo opresor (rojo) de $14 \mathrm{~mm}$ ubicados a $180^{\circ}$ uno con respecto al otro.

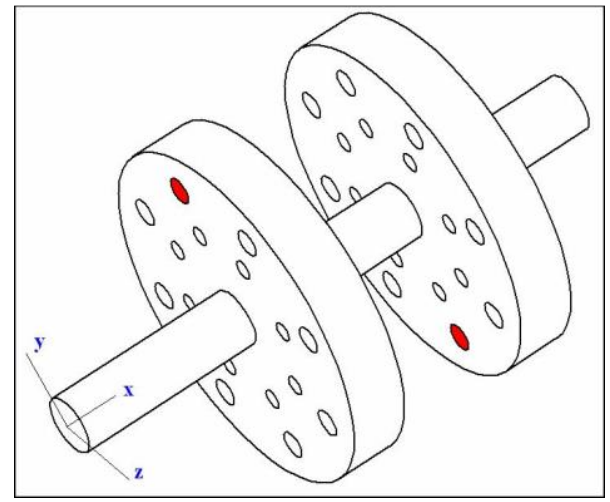

Fig. 6. Desbalance par.

La figura 7 muestra el desequilibrio dinámico, el cual se origina por la instalación de un tornillo prisionero (rojo) de $14 \mathrm{~mm}$ en cada volante, ubicados $135^{\circ}$ uno con respecto al otro.

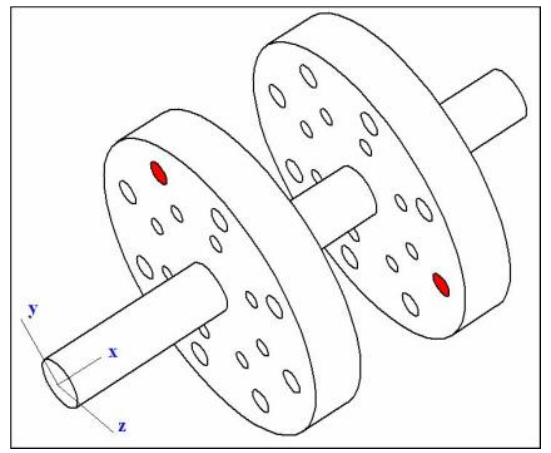

Fig. 7. Desbalance dinámico.

El diseño de estos volantes permite crear una gran cantidad de desequilibrios, haciéndolos muy versátiles a la hora de obtener el espectro característico debido al desbalance, por ejemplo, el balance de los discos se puede conseguir colocando todos los tornillos opresores y después desbalancear el sistema al retirarlos.

\subsection{Diseño y cálculo del eje para los volantes}

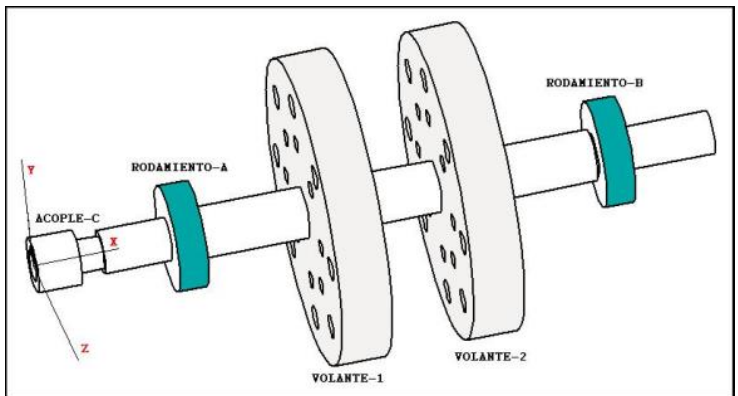

Fig. 8. Arreglo general del eje para los volantes.

En la figura 8 se observa el arreglo, el cual de ahora en adelante se llamara Eje Volante, este eje debe ser capaz de resistir todos los esfuerzos generados en el normal funcionamiento del banco.

Selección del material eje volante. Se escoge un acero AISI 1040 debido a sus propiedades de resistencia, rigidez, durabilidad, ductilidad, y facilidad relativa al maquinado. En el cuadro 14, se describe las características más relevantes de este acero.

Tabla 2. Acero AISI 1040.

\begin{tabular}{|c|c|c|c|c|}
\hline ALEACIÓN & TRATAMIENTO & $\begin{array}{l}\text { PROPIEDADES } \\
\text { MECÁNICAS }\end{array}$ & $\begin{array}{c}\text { PROPIEDADES } \\
\text { FÍSICAS Y } \\
\text { OUÍMICAS }\end{array}$ & $\begin{array}{l}\text { APLICACIONES } \\
\text { Y FORMAS }\end{array}$ \\
\hline $\begin{array}{l}1040 \\
\text { M. carbono } \\
\text { Manganeso }\end{array}$ & $\begin{array}{l}\text { Estirado en } \\
\text { frío }\end{array}$ & 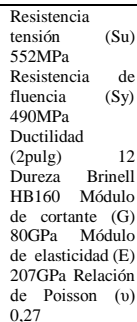 & $\begin{array}{l}\text { Densidad } 7680 \\
\mathrm{Kg} / \mathrm{m} 3 \\
\text { Coeficiente de } \\
\text { dilatación térmica } \\
(\varphi) 6,5 \times 10-6^{\circ} \mathrm{F}-1\end{array}$ & $\begin{array}{l}\text { Ejes y engranes } \\
\text { Llamado también } \\
\text { no resulfurizado }\end{array}$ \\
\hline
\end{tabular}

Fuente: (Mott, Robert, 2006), (Hamrock, Bernard, 2000).

Geometría del eje volante. El detalle constructivo de la geometría de este eje se ve en la figura 40 , donde se puede distinguir varias de las dimensiones que fueron discutidas anteriormente, como la longitud del eje, la ubicación de los soportes (rodamientos) y el posicionamiento de los volantes. También es importante resaltar en esta figura tres escalones, contra los cuales se asientan el acople, los cojinetes rodantes, y los volantes de desbalance.

En cada cambio de diámetros D1, D2 y D3, es decir en los escalones, hay dos radios de chaflanes (o radio de tangencia) para minimizar la concentración de esfuerzos; r1 y r2 son chaflanes de categoría 
agudo y redondeado respectivamente (Estupiñan, Edgar y Saavedra, Pedro, 2000). Además la transmisión de potencia desde motor hacia el eje, se realiza mediante una cuña entre el cubo del acople y el eje, lo cual se muestra en un extremo de la figura 9.

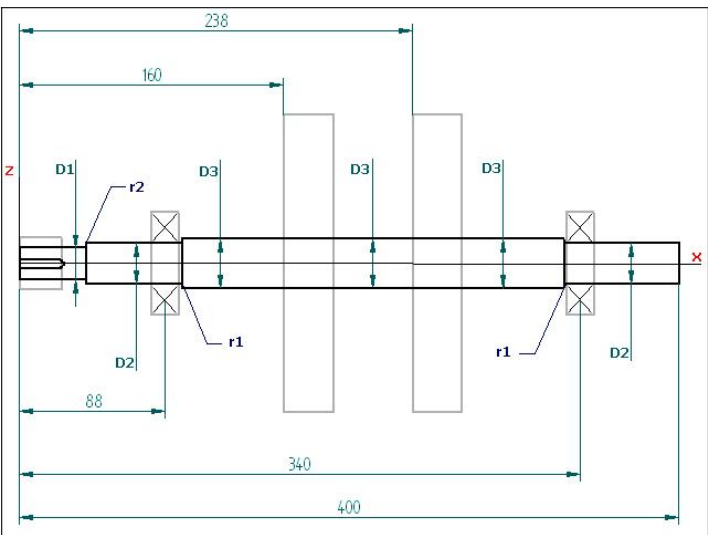

Fig. 9. Dimensiones propuestas para el eje volante (medidas en $\mathrm{mm}$ ).

Para determinar las dimensiones D1, D2, D3, r1 y r2 se deben considerar las cargas provocadas por desbalance y desalineamiento, y de esta manera obtener un diseño seguro y confiable para todo tipo de esfuerzos.

Peso de los volantes. Se calculará el peso de ambos volantes como una carga distribuida, suponiendo que estos discos son cilindros sólidos, es decir, que no poseen ningún tipo de agujero, los cuales pueden ser calculados con las ecuaciones 1 y 2 :

$D E=\frac{C E\left[N_{B} \operatorname{Cos} \gamma+G E^{\prime} \operatorname{Sen} \gamma\right]}{\left[N_{B}+C E \operatorname{Sen} \gamma\right]}$

$\alpha=\operatorname{Sen}^{-1} \frac{b}{C E}$

Teniendo en cuenta los arreglos anteriormente mencionados, es posible calcular trigonométricamente las siguientes variables:

$\rho_{\mathrm{A}}=2732 \mathrm{~kg} / \mathrm{m}^{3}$

$\mathrm{r}_{\mathrm{o}}=0,09 \mathrm{~m}$

$\mathrm{Av}=0,02545 \mathrm{~m}^{2}$

$\mathrm{Wv}=681,31 \mathrm{~N} / \mathrm{m}$

Fuerza centrífuga. Para conseguir la fuerza centrífuga generada en los discos, se deben mantener las siguientes consideraciones.

Consideraciones:
Los agujeros y los tornillos están uniformemente distribuidos en el volante.

El centro de rotación $\mathrm{CR}$ y el centro del eje CE coinciden en el mismo punto, es decir que se desprecia la deflexión del eje.

Se considera el volante como un disco sólido de aluminio con agujeros; estos espacios son dejados por tornillos prisioneros que se retiren, es decir no se tiene en cuenta la masa de estos tornillos de acero. (Ver figura 9)

Para determinar la fuerza centrífuga se utilizara el concepto radio de giro, para esto se define los ejes de referencias para el volante en el plano $\mathrm{y}-\mathrm{z}$ (Ver figura 10) esto se observa en la figura 11, en donde hay dos ejes, el primero es y'-z' que es ubicado en el centro de rotación CR y el otro es y" $-z$ " que se encuentra en el centro de masa CM; ambos ejes están separados por e, que es la excentricidad debido al desbalance originado. Además en esta figura el radio de giro con respecto a el eje x" es $\mathrm{k}-\mathrm{x}$ ", distancia en la cual se concentra toda la masa del volante independiente de cuantos agujeros pueda tener (Girdhar, Paresh, 2004).

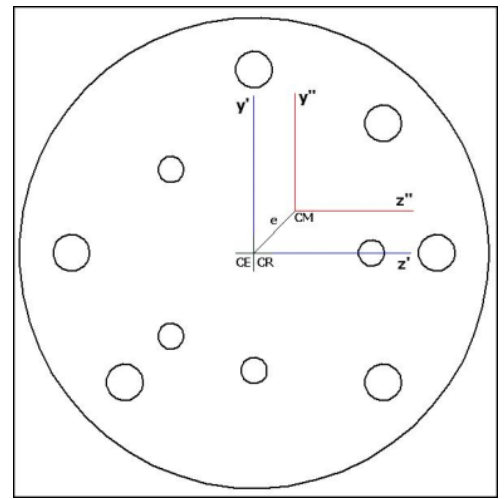

Fig. 10. Ejes de referencia para el volante.

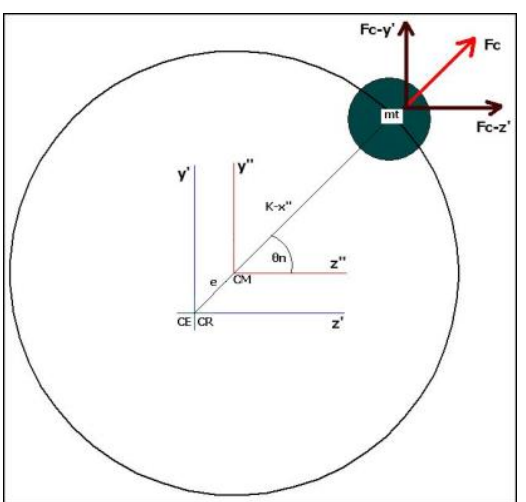

Fig. 11. Fuerza centrífuga del volante. 
Para calcular la fuerza centrífuga, se utilizan las siguientes ecuaciones 3-8:

$$
\begin{aligned}
& F_{C}=\frac{m\left(k-x^{\prime \prime}+e\right) w^{2}}{1000} \\
& k-x^{\prime \prime}=\sqrt{\frac{I x^{\prime \prime}}{m}} \\
& w=\frac{2 \pi}{60} n \\
& e=\sqrt{\left(y_{C}\right)^{2}+\left(z_{C}\right)^{2}} \\
& F c_{y^{\prime}}=\left(F_{C}\right) \operatorname{Sen} \theta_{n} \\
& F c_{z^{\prime}}=(F c) \operatorname{Cos} \theta_{n}
\end{aligned}
$$

El ángulo para ubicar la fuerza centrífuga $\theta$ n se encuentra con la ayuda del centro de masa CM, este ángulo cambia debido a la configuración del volante debido a que depende de cuantos tornillos se coloquen o cuantos se retiren, como se presenta en la siguiente ecuación 9:

$$
\theta_{1}=\operatorname{Tan}^{-1}=\frac{y_{C}^{\prime}}{z_{C}^{\prime}}
$$

Para localizar adecuadamente la fuerza centrífuga se necesita tener en cuenta las siguientes condiciones:

$\operatorname{Si}\left(\mathrm{zc}^{\prime}=0\right), \theta 1=90^{\circ} \mathrm{y} \mathrm{si}\left(\mathrm{yc}^{\prime}=0\right), \theta 1=0^{\circ}$

$\operatorname{Si}\left(\mathrm{yc}^{\prime}>0\right)$ y $\left(\mathrm{zc}^{\prime}>0\right)$, entonces $\theta \mathrm{n}=\theta 1$

Si $\left(\mathrm{yc}^{\prime}>0\right)$ y $\left(\mathrm{zc}^{\prime}<0\right)$, entonces $\theta \mathrm{n}=\theta 2=180-|\theta 1|$

Si $\left(\mathrm{yc}^{\prime}<0\right)$ y $\left(\mathrm{zc}^{\prime}<0\right)$, entonces $\theta \mathrm{n}=\theta 3=180+|\theta 1|$

Si $\left(\mathrm{yc}^{\prime}>0\right)$ y $\left(\mathrm{zc}^{\prime}<0\right)$, entonces $\theta \mathrm{n}=\theta 4=360-|\theta 1|$

Si $\left(y c^{\prime}=0\right)$ y $\left(z^{\prime}=0\right), e=0$ lo cual significa que la fuerza centrífuga debido al desbalance no exista, pero como se considera el concepto de radio de giro entonces la fuerza centrífuga sería simplemente por el peso del volante, ya que k-x"' es diferente de cero.

Debido a que el centro de masa varia con la cantidad de tornillos prisioneros instalados en el volante para obtener el centroide (yc' - zc'), la masa total (mt) y el momento de inercia centroidal (Ix') para cualquier combinación posible.

La ecuación 11 es modificada, para obtener un valor más real de esta fuerza, incluyendo la deflexión del eje, con longitud igual a $400 \mathrm{~mm}(\delta=1,2 \mathrm{~mm})$ (Mott, R. 2006), donde:

$F_{C}=\frac{m\left(k-x^{\prime \prime}+e+\delta\right) w^{2}}{1000}$

Revisión de la deflexión del eje volante. Es necesario analizar la deflexión del eje tanto a torsión como a flexión, para esto se utiliza las siguientes recomendaciones para los límites de estas deflexiones:

- Deflexión debido a flexión: 0,2mm para partes de máquinas en general

- Deflexión (rotación) debido a la rotación: 0,016 para partes de máquinas

La deflexión debido a la torsión se calcula mediante las siguientes ecuaciones 12 y 13, cuyos resultados se muestran en la Tabla 3.

$\theta=\frac{\mathrm{T}(\mathrm{L})}{\mathrm{G}(\mathrm{J})}$

$J=\frac{\pi\left(d^{4}\right)}{32}$

Tabla 3. Deflexión debido a la torsión.

\begin{tabular}{cccccc}
\hline \multirow{2}{*}{$\mathrm{T}_{2}\left(\frac{\mathrm{N}}{\mathrm{mm}}\right)$} & $\mathrm{G}\left(\frac{\mathrm{N}}{\mathrm{mm}^{2}}\right)$ & $\mathrm{L}(\mathrm{mm})$ & $\mathrm{D}(\mathrm{mm})$ & $\mathrm{J}\left(\mathrm{mm}^{4}\right)$ & $\theta(\mathrm{rad})$ \\
\hline \multirow{4}{*}{2430} & & 40 & 22 & 22998,02 & $5,28 \times 10^{-5}$ \\
& 18 & 30 & 79521,56 & $6,87 \times 10^{-5}$ \\
& 45 & 35 & 147323,5 & $9,38 \times 10^{-5}$ \\
& 13 & 43 & 335640,0 & $1,22 \times 10^{-5}$ \\
& 194 & 45 & 402577,9 & $1,46 \times 10^{-5}$ \\
& 13 & 43 & 335640,0 & $1,22 \times 10^{-5}$ \\
& 45 & 35 & 147323,5 & $9,38 \times 10^{-5}$ \\
& 30 & 30 & 79521,5 & $1,14 \times 10^{-5}$ \\
& $\sum \theta$ & & & 0,00613 \\
\hline
\end{tabular}

Donde:

$\mathrm{T}_{2}=$ Torque eje volante

$\mathrm{d}=$ Diámetro del eje en cada sección.

$\theta=$ Deflexión torsional.

$\mathrm{L}=$ Longitud de la cada sección eje.

$\mathrm{G}=$ Módulo cortante.

$\mathrm{J}=$ Momento de inercial polar.

$\Sigma \theta=$ Deflexión del eje.

Al comparar la recomendación en cuanto al límite de la deflexión debida a torsión con la calcula en la tabla 3 , se observa claramente que el diseño es confiable ya que $\Sigma \theta\left(0,006^{\circ}\right)$ es menor a $0,016^{\circ}$.

La deflexión debido a flexión del eje para los planos $(\mathrm{y}-\mathrm{x})$ y $(\mathrm{z}-\mathrm{x})$ se muestran en las figuras 12 y 13. Las 
cuales determinaron mediante integración de las siguientes ecuaciones 14 y 15 .

$$
\begin{gathered}
\frac{d^{2} y}{d x^{2}}=\frac{M_{y}}{E I} \\
\frac{d^{2} z}{d x^{2}}=\frac{M_{z}}{E I}
\end{gathered}
$$

Donde:

$\mathrm{M}(\mathrm{z}$ o y $)=$ Momento, $\mathrm{N}-\mathrm{mm}$.

$\mathrm{E}=$ Modulo de elasticidad $207 \times 103 \mathrm{~N} / \mathrm{mm}^{2}$.

$\mathrm{I}=$ Momento de inercia $(\pi(\mathrm{d} 4)) / 64)$.

$\mathrm{d}=$ Diámetro del eje en cada sección, $\mathrm{mm}$.

La deflexión máxima debida a flexión se determina mediante la siguiente ecuación 16 :

$\partial=\left(\partial_{\mathrm{z}}\right)^{2}+\left(\partial_{\mathrm{y}}\right)^{2}$

Donde

$\partial_{\mathrm{z}}=$ Deflexión máxima en el plano $\mathrm{z}$

$$
-\mathrm{x}, \mathrm{mm},(8,172 \mathrm{x} 10-2 \mathrm{~mm})
$$

$\partial_{\mathrm{y}}=$ Deflexión máxima en el plano y

$$
-\mathrm{x}, \mathrm{mm},(10,671 \mathrm{x} 10-2 \mathrm{~mm})
$$

Entonces la deflexión máxima es $\partial=13,44 \times 10-$ $3 \mathrm{~mm}$

Comparando la recomendación antes citada para esta deflexión se observa que $0,13 \mathrm{~mm}$ es menor a $0,2 \mathrm{~mm}$, por lo tanto el diseño resulta confiable (Ferri, Mario, 2008).

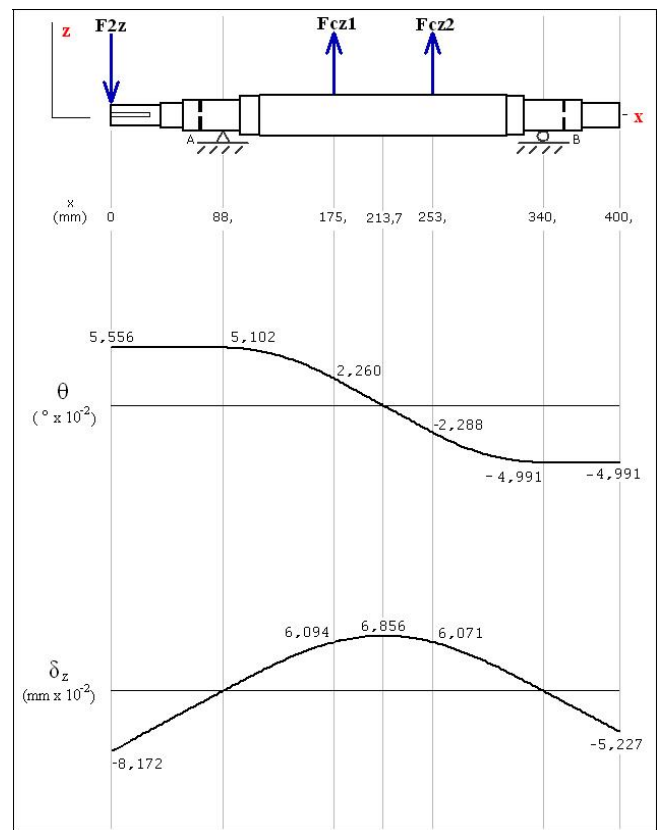

Fig. 12. Diagrama de pendiente y deflexión eje volante, en el plano $z-x$.

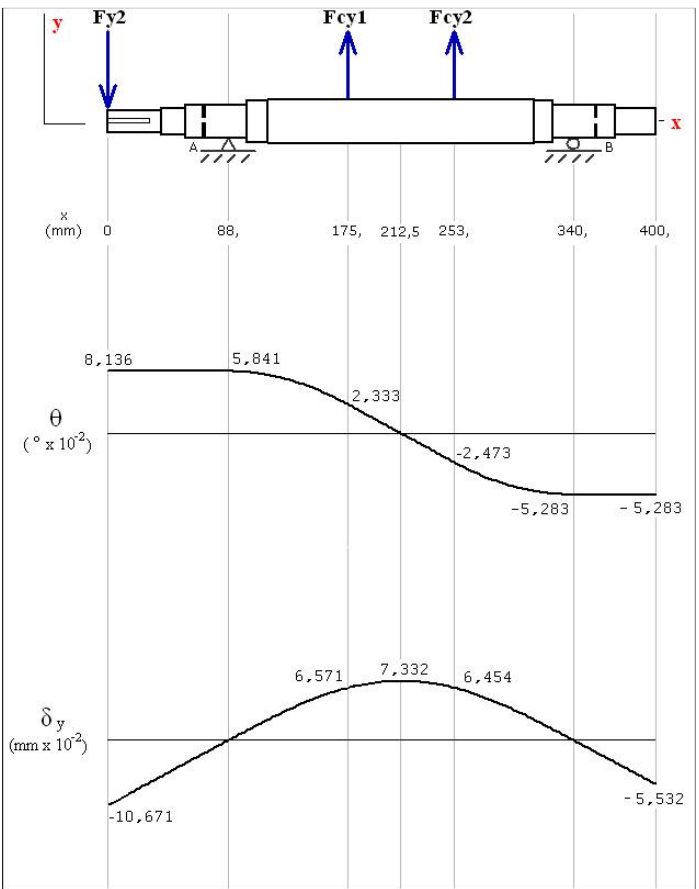

Fig. 13. Diagrama de pendiente y deflexión eje volante, en el plano $y-x$.

Cálculo de la vibración. Aunque el banco de pruebas estará destinado a generar vibraciones, es necesario revisar la vibración lateral y torsional del eje. El objetivo es calcular la frecuencia natural o fundamental del eje y compararla con la de funcionamiento.

Vibración lateral: Para determinar esta vibración se utilizara dos métodos aproximados.

Método de Rayleigh, donde la frecuencia natural es igual a la siguiente ecuación 17 :

$\mathrm{W}_{\mathrm{n}}=\sqrt{\mathrm{g} \frac{\sum_{\mathrm{i}=1}^{\mathrm{n}} \mathrm{W}_{\mathrm{i}} \mathrm{O}_{\mathrm{i}}}{\sum_{\mathrm{i}=1}^{\mathrm{n}} \mathrm{W}_{\mathrm{i}} \mathrm{Q}_{\mathrm{i}}}}$

Para conocer el resultado de la ecuación 17, se necesita calcular los pesos. Peso acople, donde $\mathrm{ma}_{1}=\mathrm{ma}_{2}, \mathrm{yma}$.

$\mathrm{W}_{1}=\frac{\text { Wacople }}{2}=0,5\left(\mathrm{ma}_{1}+\mathrm{ma}_{2}+\mathrm{ma}_{3}\right) \mathrm{g}$

$\mathrm{W}_{1}=0,5(0,58(2)+2,065)(9,8)=16 \mathrm{~N}$

Peso volante 1 y 2 , donde $\mathrm{W}_{\mathrm{v} 1}=\mathrm{W}_{\mathrm{v} 2}$ ecuación 3.17 , y hat $=0,03 \mathrm{~m}$. 
$\mathrm{W}_{2}=\mathrm{W}_{\text {volante } 1}=\mathrm{W}_{\mathrm{v} 1} *$ hat

$\mathrm{W}_{2}=(681,31 \mathrm{~N} / \mathrm{m})(0,03 \mathrm{~m})=21 \mathrm{~N}$

$\mathrm{W}_{3}=\mathrm{W}_{\text {volante1 }}=\mathrm{W}_{\text {volante } 2}$

También se requiere de las deflexiones cuando los dos volantes $\left(\mathrm{W}_{2}-\mathrm{W}_{3}\right)$ y el acople $\left(\mathrm{W}_{1}\right)$ están instalados en el eje:

$\delta 1=$ Acople $=0,0003999 \mathrm{~mm}$

$\delta 2=$ Volante $1=0,0001216 \mathrm{~mm}$

$\delta 3=$ Volante $2=0,0001491 \mathrm{~mm}$

Ahora se determina la frecuencia natural con la ecuación 17 de la siguiente manera:

$W_{n}=\sqrt{\frac{16(0,0003999)+21(0,0001216)+21(0,0001491)}{16(0,0003999)^{2}+21(0,0001216)^{2}+21(0,0001491)^{2}}}$

$\mathrm{W}_{\mathrm{n}}=5958 \mathrm{rad} / \mathrm{s}$

$\mathrm{n}_{\mathrm{C}}=\frac{2 \pi}{60} \mathrm{~W}_{\mathrm{n}}=56893 \mathrm{rpm}$

Método de Dunkerley: Donde la frecuencia natural es igual a:

$\frac{1}{\left(W_{n}\right)^{2}}=\frac{1}{\left(W_{n 1}\right)^{2}}+\frac{1}{\left(W_{n 2}\right)^{2}}+\frac{1}{\left(W_{n 3}\right)^{2}}$

Para este método, se necesita la deflexión cuando cada elemento esta instalados en el eje de forma independiente, por ejemplo para la deflexión del acople se determina sin ningún otro elemento instalado.

$\delta 1=$ Acople $=0,0007301 \mathrm{~mm}$

$\delta 2=$ Volante $1=0,0001384 \mathrm{~mm}$

$\delta 3=$ Volante $2=0,0001384 \mathrm{~mm}$

Ahora se determina la frecuencia natural de Dunkerley mediante la ecuación 18 de la siguiente manera:

$$
\begin{aligned}
& \omega_{\mathrm{n} 1}=\sqrt{\frac{\mathrm{g}}{\partial_{1}}}=\sqrt{\frac{9800}{0,0007301}}=3664 \mathrm{rad} / \mathrm{s} \\
& \omega_{\mathrm{n} 2}=\sqrt{\frac{\mathrm{g}}{\partial_{2}}}=\sqrt{\frac{9800}{0,0001384}}=8415 \mathrm{rad} / \mathrm{s} \\
& \omega_{\mathrm{n} 3}=\sqrt{\frac{\mathrm{g}}{\partial_{3}}}=\sqrt{\frac{9800}{0,0001384}}=8415 \mathrm{rad} / \mathrm{s}
\end{aligned}
$$

$\frac{1}{\left(\omega_{\mathrm{n}}\right)^{2}}=7,449 \times 10^{-8}+2\left(1,4121 \times 10^{-8}\right)$

$\omega_{\mathrm{n}}=3120 \mathrm{rad} / \mathrm{s}$

$\mathrm{n}_{\mathrm{C}}=\frac{2 \pi}{60}(3120)=29793 \mathrm{rpm}$

Por medio de las ecuaciones anteriores se establece que la operación de funcionamiento $(1800 \mathrm{rpm})$ es mucho menor a estas velocidades críticas (2979356893rpm), lo cual evita el fenómeno de resonancia (Elesaganter. Solid handwheels, 2013).

Vibración torsional: De la misma forma que el eje puede vibrar lateralmente, actuando como resortes a flexión, también lo hace torsinalmente, con una o más frecuencias naturales a torsión. La vibración torsional de un eje con dos discos es igual a la siguiente ecuación 19:

$\omega_{n-T}=\sqrt{\frac{I_{1}+I_{2}}{\left(I_{1}\right)\left(I_{2}\right)}}$

Además se necesita conocer las siguientes ecuaciones 20 y 21 complementarias a la ecuación 19.

$\mathrm{K}_{\mathrm{t}}=\frac{\mathrm{JG}}{\mathrm{L}_{\mathrm{y}}}$

$\mathrm{I}=\mathrm{I}_{1}+\mathrm{I}_{2}=\frac{\mathrm{W}_{\text {Volante }}\left(\mathrm{r}_{0}\right)^{2}}{2 \mathrm{~g}}$

Las variables que intervienen en las tres ecuaciones anteriores son:

$\mathrm{I}=$ Momento de inercia del volante 2 o 1, $\mathrm{kg}$

$$
-\mathrm{m}-2
$$

$\mathrm{Lv}=$ Longitud entre volantes, 0,048 m.

ro $=$ Radio de los volantes, $0,090 \mathrm{~m}$.

$\mathrm{G}=$ Módulo de rigidez del eje, $80 \times 109 \mathrm{~N} / \mathrm{m}^{2}$.

$\mathrm{J}=$ Momento polar del eje, 4,026×10-7 $\mathrm{m}^{4}$.

$\mathrm{W}_{\text {volante }}=$ peso del volante, $21 \mathrm{~N}$

Para mayor seguridad en el resultado de esta velocidad se utilizará el momento de inercial de los volantes con todos los tornillos prisioneros instalados. De esta manera se resuelve las ecuaciones de la 19 a la 21.

$I_{1}=I_{2}=\frac{21(0.09)^{2}}{2(9.8)}=8,68 \times 10^{3} \mathrm{~kg}-\mathrm{m}^{2}$ 
$J=\frac{\pi(0.045)^{4}}{32}=4,026 \times 10^{-7}-\mathrm{m}^{4}$

$\mathrm{K}_{\mathrm{t}}=\frac{\left(4,026 \times 10^{-7}\right)\left(80 \times 10^{9}\right)}{0.048}=670963,2 \mathrm{~N}-\mathrm{m}$

$\omega_{\mathrm{n}-\mathrm{T}}=\sqrt{670963,2 \frac{2\left(8,68 \times 10^{-3}\right)}{\left(8,68 \times 10^{-3}\right)}}=12434 \mathrm{rad} / \mathrm{s}$

$\mathrm{f}_{\mathrm{n}-\mathrm{T}}=\frac{(12434) 60}{2 \pi}=118736 \mathrm{rpm}$

\subsection{Diseño tornillo desalineación horizontal}

Este tornillo se encarga de desalinear el eje de los volantes con respecto al eje del motor, y de esta manera generar las vibraciones por desalineamiento; su ubicación es debajo de cada soporte y es accionado manualmente como se observa en la figura 14. Antes de comenzar el diseño del tornillo, se debe encontrar el peso a mover, donde:

$$
\begin{aligned}
& \mathrm{W}=\mathrm{W}_{\text {Acople }}+\mathrm{W}_{\text {Eje }}+\mathrm{W}_{\text {Volante }}+\mathrm{W}_{\text {Soporte }} \\
& \mathrm{W}_{\text {Acople }}=\left(\mathrm{m}_{\mathrm{a} 1}+\mathrm{m}_{\mathrm{a} 2}+\mathrm{m}_{\mathrm{a} 3}\right) \mathrm{g} \\
& \mathrm{W}_{\text {Acople }}=[2(0.58)+2.065] 9.8=32 \mathrm{~N} \\
& \mathrm{~W}_{\text {Eje }}=\mathrm{g}(\rho) \mathrm{V}=\mathrm{g}\left(\rho_{\mathrm{s}}\right)\left(\pi \mathrm{r}^{2} \mathrm{~L}\right) \\
& \mathrm{W}_{\text {Eje }}=9.8(7680) \pi(0.0225)^{2}(0.4)=48 \mathrm{~N} \\
& \mathrm{~W}_{\text {Volante }}=2(681.31)(0.03)=42 \mathrm{~N} \\
& \mathrm{~W}_{\text {Soporte }}=2(2.6)(9.8)=51 \mathrm{~N}
\end{aligned}
$$

Teniendo en cuenta la ecuación 22 se tiene que:

$\mathrm{W}=173 \mathrm{~N}$

Para mayor confiabilidad se utiliza un factor de seguridad igual a 3,41 , de esta manera se obtiene lo siguiente:

$W_{d}=3,41 W=3,41(173)=590 \mathrm{~N}$

Además de esta carga se debe establecer el desplazamiento máximo de los dos tornillos, y su movimiento relativo uno con respecto a otro, conservando las condiciones de desalineamiento permisible.
Esto se observa en la figura 63, la cual muestra el desalineamiento esperado en los soportes al manipular cada tornillo. Al mover $30 \mathrm{~mm}$ el soporte A y $20 \mathrm{~mm}$ el soporte $\mathrm{B}$, se origina una desalineación angular de $13^{\circ}$ y $2^{\circ}$ para el acople y rodamientos respectivamente, lo cual está dentro de los valores permitidos de diseño. También es necesario aclarar que el desplazamiento máximo del elemento intermedio del acople $(151,97 \mathrm{~mm})$ se mantiene dentro de los límites (Harris, Cyril, 2002) (A-MAQ, 2005). Las vibraciones de desalineamiento en el banco se manifiestan con las siguientes combinaciones de movimientos máximos del tornillo:

- Desalineamiento Angular: Mover el soporte A $10 \mathrm{~mm}$

- Desalineamiento Paralelo: Mover el soporte A $20 \mathrm{~mm}$ y soporte B $20 \mathrm{~mm}$

- Combinación desalineamiento: Mover el soporte A $30 \mathrm{~mm}$ y soporte B $20 \mathrm{~mm}$

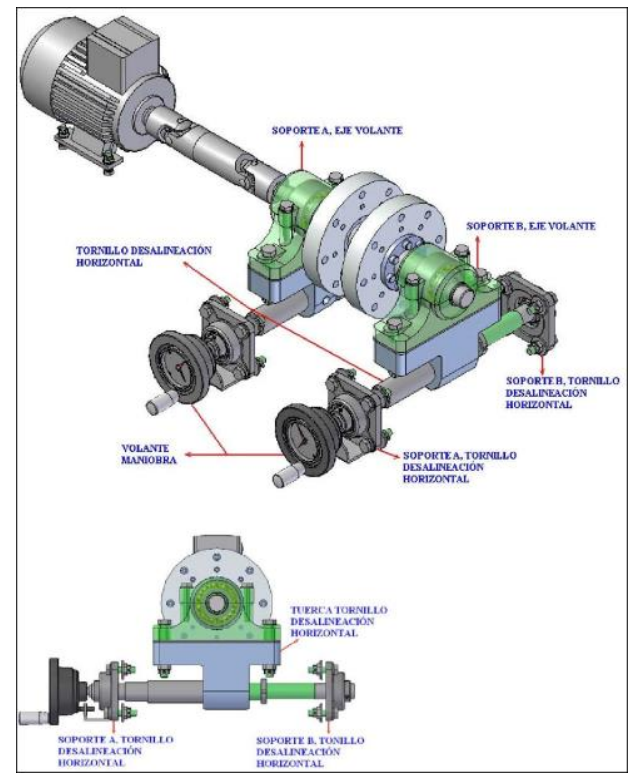

Fig. 14. Desalineamiento horizontal del banco de prueba.

\section{CONCLUSIONES}

Se concluye la importancia que tiene para el ingeniero mecánico el conocimiento e identificación de las vibraciones originadas por desbalance, desalineamiento y defectos en rodamientos, lo que permite estudiar la respuesta dinámica que cada componente ante estas manifestaciones. Se realizó un investigación teórica sobre este tema, la cual es muy escasa en español pero en ingles se puede conseguir buena información. 
Se logró diseñar un sistema de desalineación vertical y horizontal mediante tres tornillos de rosca métrica, de manipulación sencilla, ergonómicos, debidamente localizados con la posibilidad de reproducir dos tipos de desalineamiento el paralelo y el angular o una combinación de ambos, y con indicadores que proporcionan una medida exacta de sus respectivos desplazamientos; además dos discos de aluminio con tornillos prisioneros que originan el desequilibrio deseado, lo cuales se fijan al eje mediante ensambles seguros que facilitan el montaje y desmontaje.

También se pudo determinar el tamaño del eje, el cual estará destinado a soportar todas las cargas y esfuerzos presentes durante la operación normal del banco. Esto permitió seleccionar los soportes, los rodamientos y el acople más apropiado para muchas condiciones de funcionamiento.

Se estableció un sistema eléctrico confiable capaz de arrancar, detener y variar la velocidad de un motor trifásico de jaula de ardilla de medio caballo, con protección y corte de energía ante eventuales problemas, todo esto encerrado en un gabinete, cuyo diseño ofrece maniobra segura para cualquier persona y una buena transferencia de calor por convección natural a sus dispositivos internos. De igual forma un sistema electrónico preciso que captura las señales vibratorias de cada soporte, y un software programable que visualiza, la onda temporal y el espectro de aquellos defectos generados por el banco.

Con la implementación de una estructura estable y sus respectivos aislantes, se obtuvo un banco rígido, con muy poca transmisibilidad de aquellas oscilaciones que son provocadas por el banco hacia los instrumentos eléctricos.

Se verificó el diseño del banco de pruebas mediante un análisis de elementos finitos, lo que demostró la confianza de la fuerza centrífuga y el concepto de radio de giro en los cálculos de diseño correspondientes a algunos elementos mecánicos que hace parte de este proyecto, por lo tanto la fuerza dinámica máxima esperada durante la operación se mantiene dentro de lo permisible, tanto para el eje, soportes y rodamientos. De la misma manera se comprobó los movimientos de cada componente para evitar rozamientos inesperados.

Como se observa en este diseño la mayoría de los componentes del banco se sobredimensionaron, ya que se consideró la ambigüedad de las cargas dinámicas cuando exista una combinación no estimada, de desalineamiento, desequilibrio y fallas en rodamientos, lo cual mejora ostensiblemente la respuesta de estos elementos a una operación severa, que algunas veces puede llegar a ser desconocida.

Para este trabajo de grado una pieza delgada o pequeña según sea el caso, provocaría que nuestro sistema vibre descomunalmente, debido a que la masa puede absorber tales vibraciones se resolvió que estos dispositivos fuesen más robustos que lo normal, como por ejemplo la estructura del banco.

Se implantó un sistema de lubricación económico y eficiente para cada tornillo de desalineamiento, una excelente fijación entre uniones mediante tuercas ranuras y pasadores lo que impiden aflojamientos indeseados, y una transmisión manual efectiva por medio de poleas sincrónicas que levantan el motor y produce un desalineamiento vertical.

Se diseñó un banco de pruebas para fines didácticos de fácil manejo, seguro para cualquier operador, de fácil mantenimiento, factible y viable desde el punto de vista tecnológico; destinado a estudiar la teoría de falla para máquinas rotativas

mediante el análisis de vibraciones mecánicas, referido a problemas dedesbalance o desequilibrio, desalineamiento $\mathrm{y}$ grietas $\mathrm{o}$ defectos en los componentes de un rodamiento, utilizando como herramienta esencial el espectro de frecuencia; el cual se caracteriza primordialmente porque todos sus componentes (mecánicas, eléctricas y electrónicos) son estándares, relativamente fáciles de adquirir o /y fabricar según sea el caso; además con buenas recomendaciones de ajuste y tolerancia para un correcto montaje y unexcelente funcionamiento.

\section{REFERENCIAS}

Advanced Antivibration Components. (2004). Shock and vibration damping components catalog. [En línea]. Canada: vibrationmounts, [citado 23 junio 2018] Disponible en Internet: < http://www. Vibrationmounts.com/V100/PDF/Download /V100.zip>

A-MAQ. (2005). Análisis de maquinaria. Tutorial de vibraciones para mantenimiento mecánico. [En línea]. Medellín: a-maq. [Citado 11 de enero de 2018]. Disponible en internet: < http://www.a-maq.com/ tutoriales/Tutorial\%20Vibraciones $\% 20$ para 
$\%$ 20Mantenimiento \%20Mecanico\% 20AMAQ\%202005.pdf>

Carrero-Blanco, J., Estrems, S., \& Sánchez, H. (2016). Método semianalítico para la mecánica del contacto entre el eje y la chumacera en un vehículo sumergible. Revista Internacional de Métodos Numéricos Para Cálculo y Diseño En Ingeniería, 32(4), 240-251.

https://doi.org/10.1016/j.rimni.2015.07.003

Dytran Instruments INC. (2007). Specifications industrial accelerometer. Model 3185D. [en línea]. España: Dytran, 2007. [Citado en julio 2018]. Disponible en Internet: http://www.dytran.com/img/products/3185 D.pdf

E Florez, CAP Cortes. (2017), Aplicación del método de la ecuación de Boltzmann en redes para la simulación bidimensional de un problema típico de mecánica de fluidos Revista Colombiana de Tecnologías de Avanzada ISSN: 1692-7257.

Elesaganter. Solid handwheels. (2013). [En línea]. España: elesa-ganter. [Citado 22 marzo 2018] Disponible en Internet: http://www.elesaganter.com/scheda_es_2_7 696_20_2.aspx

Estupiñan, Edgar y Saavedra, Pedro. (2000). Técnicas de diagnóstico para el análisis de vibraciones de rodamientos. [En línea]. Chile: mantenimiento planificado. [Citado 12 abril de 2018] Disponible en Internet:< http:// www.mantenimientoplanificado.com/ artículos\% 20PREDICTI VO_ archivos/ galileo.dim. Edec.cl\%

201mv/articulo_rodamientos_CHILE1.pdf>

Ferri, Mario. (2008). Universal joints. Diagram for joints series H. [en línea]. España: marioferri. -[citado 12 Mayo de 2018] Disponible en internet: http://www.marioferri.com/page_ing/main/p df/diagramma_H.pdf

FEM García, JJ Ramirez-Mateus..., (2017), SISTEMA DE CONTROL ACTIVO RESONANTE INTEGRAL PARA VIBRACIONES EN UNA VIGA EN VOLADIZO Revista Colombiana de Tecnologías de Avanzada ISSN: 1692-7257
García-León, R. A., Flórez-Solano, E. N., \& Acosta, M. A. (2015). Analisis estructural de una maquina prensadora para la producción de ladrillo macizo para las pequeñas industrias artesanales de materiales cerámicos en Ocaña Norte de Santander y la región. Revista Colombiana de Tecnologías de Avanzada, 1(25), 104-109.

García-León, R. A., Flórez-Solano, E. N., \& Escobar-Macea, M. (2016). Modelo de equipo de prensado tipo palanca, para mejorar la producción de queso en la provincia de Ocaña. Revista Colombiana de Tecnologías de Avanzada, 2(28), 140-149.

García-León, R. A., Flórez-Solano, E. N., \& Sanchez-Ortiz, E. (2017). Diseño de un sistema alimentador para un horno rotatorio en la producción de fosfato en el Norte de Santander. Revista Colombiana de Tecnologías de Avanzada, 1(29), 10-80.

Girdhar, Paresh. (2004). Practical machinery vibration analysis and predictive maintenance. New York: Newnes. 91p

González, Gonzalo. (2003). Transmisión por correa. [En línea]. España: elprisma. [Citado 24 febrero de 2018]. Disponible en Internet: < http:// www. elprisma.com /apuntes/ ingenieria

_mecanica/transmisioncorrea/default.asp>

Hamrock, Bernard. (2000). Elementos de máquinas. México: McGraw Hill. 131 p.

Harris, Cyril. (2002). Shock and vibration handbook. New York: McGraw Hill. 190 p.

JES Jaimes, JAV Ochoa. (2017). ANÁLISIS DE CRITICIDAD Y ÁRBOLES DE DIAGNÓSTICO DE FALLAS PARA TRANSFORMADORES DE POTENCIA Revista Colombiana de Tecnologías de Avanzada ISSN: 1692-7257.

Mott, Robert. Diseño de elementos de máquinas. México: Prentice Hall, 2006. p.6. 